\title{
Effect of gravity on the stability of viscoelastic thermocapillary liquid layers
}

\author{
Kai-Xin $\mathrm{Hu}^{\mathrm{a}, *}$, Meng $\mathrm{He}^{\mathrm{b}}$, Qi-Sheng Chen ${ }^{\mathrm{b}, \mathrm{c}}$, Rong Liu ${ }^{\mathrm{d}}$ \\ a School of Mechanical Engineering and Mechanics, Ningbo University, Ningbo, Zhejiang 315211, China \\ ${ }^{\mathrm{b}}$ School of Engineering Science, University of Chinese Academy of Sciences, Beijing 100190, China \\ 'Key Laboratory of Microgravity, Institute of Mechanics, Chinese Academy of Sciences, Beijing 100190, China \\ ${ }^{\mathrm{d}}$ School of Mechanical and Electrical Engineering, Gui Lin University of Electronic Technology, Gui Lin 541004, China
}

\section{A R T I C L E I N F O}

\section{Article history:}

Received 27 April 2017

Received in revised form 11 February 2018

Accepted 22 February 2018

Available online 20 March 2018

\begin{abstract}
A B S T R A C T
Thermocapillary convection appears in many polymer processing operations. In order to understand the effect of gravity for its stability, three-dimensional linear stability analysis is performed for a viscoelastic fluid. The critical Marangoni number is derived as a function of Bond number, Prandtl number and elasticity. When the Prandtl number is large, the increasing of gravity effect often makes the flow more stable, and the coupling of gravity to elasticity and thermocapillary force excites many different kinds of preferred modes. For small Prandtl number, the flow is always destabilized by gravity effect. The work done by gravity becomes a new energy source for perturbation. However, it can be either positive or negative, which is not directly related to the variation of critical Marangoni number with gravity. The effect of gravity on the instability mechanism and the properties of preferred modes are demonstrated for different elasticity.
\end{abstract}

(c) 2018 Elsevier Ltd. All rights reserved.

\section{Introduction}

Thermocapillary flow refers to the surface-tension-driven convection in the fluid layer submitted to a horizontal temperature gradient. It has been an active topic of research for its great practical importance in many industrial applications, such as fusion welding [1] and crystal growth techniques [2]. In the theoretical studies, the model proposed by Smith \&Davis [3] has been widely used, where the fluid layer above an infinite flat plane is set in motion by the a horizontal temperature gradient on the surface. The oblique hydrothermal waves predicted in their work have been observed both in the experiment [4] and numerical simulation [5].

This model has also been used in the study of thermocapillary flow in the presence of gravity. Garr-Peters [6] has performed linear stability analysis for surface-tension driven fluid layer subject to buoyant forces. The free surface is facing either upward or downward while the Prandtl numbers considered include $0.01 \leqslant \operatorname{Pr} \leqslant 10$. It was shown that the gravity destabilizes the flow for small Prandtl numbers. Parmentier et al. [7] have examined the stability of coupled buoyancy and thermocapillary driven convection in thin fluid layers for $0.01 \leqslant P r \leqslant 7$. The presence of travelling rolls is exhibited. Mercier and Normand [8] have studied the linear

\footnotetext{
* Corresponding author.

E-mail address: hukaixin@nbu.edu.cn (K.-X. Hu).
}

stability of buoyant-thermocapillary liquid layers for $\mathrm{Pr}=7$. The transition between stationary and oscillatory modes found in the experiment of Daviaud and Vince [9] is observed when the heat transfer at the free surface is introduced. Chan and Chen [10] have carried out linear stability analysis of the thermocapillary fluid layer with the effect of gravity included for $\mathrm{Pr}=13.9$. The results compare favorably with the experiment conducted by Riley and Neitzel [4]. The critical Marangoni number increases with Grashof number (a measure of gravity) while the preferred mode changes from oblique wave to transverse wave.

It should be noted that the model used above is very different from the Benard-Marangoni convection [11] although gravity and thermocapillary forces exist in both of them. For the latter, the fluid layer is heated from below. However, for the former, the bottom has zero heat flux. A horizontal temperature gradient is imposed on the fluid surface, and there is an inclined temperature gradient with both a horizontal and a vertical component in the fluid layer.

The thermocapillary flows for polymer liquids have also received much attention for its applications in film coating [12], drying of polymer solution [13,14], dewetting [15] and polymer processing $[16,17]$. It is worth noting that polymer liquids are often viscoelastic fluids, whose flow properties are very different from those of Newtonian fluids. The effect of elasticity should be considered in the study of thermocapillary flows for polymer liquids. 
A few investigations have been undertaken to study the stability of viscoelastic thermocapillary liquid layers. The influence of thermocapillary forces on the buoyancy-driven viscoelastic fluid layer has been demonstrated by Kaloni \& Lou with Prandtl numbers 10 and 100 [18]. The thermocapillary force is far less than the buoyancy force and the perturbations they considered are mainly transverse and longitudinal modes. Davalos-Orozco \& Chavez [19] have performed two-dimensional linear stability analysis in small wave number approximation for thermocapillary convection in a viscoelastic fluid layer under a horizontal temperature gradient. Tong et al. [20] have investigated the thermocapillar instability of a two-dimensional viscoelastic planar liquid sheet in surrounding gas. Hernández and Dávalos-Orozco [21] have examined the linear viscoelastic thermal Marangoni convection. Gravity has been neglected and the system is heated from the lower wall. The competition between stationary and oscillatory convection is shown. Hu et al. [22] have studied the instability of thermocapillary liquid layers for Oldroyd-B fluid. Three kinds of preferred modes are found in different elasticity: oblique wave, streamwise wave and spanwise stationary mode. For the first, the flow is stabilized by elasticity. The second has several fluctuations in vertical direction. The last becomes the preferred mode when the elasticity is high enough.

The impact of elasticity on flow stability has been demonstrated in the above works. However, the ground experiment and processing of polymer liquids are often in a normal gravity environment. The works for thermocapillary convection of Newtonian fluid suggest that the gravity has a significant impact on the flow stability $[4,10]$. Thus, the clarification of gravity effect in viscoelastic thermocapillary convection is also needed, which is the purpose of this paper.

In the present work, the study of gravity effect on the stability of thermocapillary convection has been extended from Newtonian fluid [10] to viscoelastic fluid. The Oldroyd-B fluid is applied, which is a viscoelastic model widely used for dilute solutions of macromolecules. In order to show the coupling effect of gravity, elasticity and thermocapillary force, the flow at several Bond numbers, elastic numbers and Prandtl numbers are examined. The comparisons are made with the case without gravity [22] and the effect of gravity on the instability mechanism is discussed.

\section{Problem formulation}

We consider the model of thermocapillary liquid layers presented by Smith and Davis [3], where the fluid on an infinite wall is set in motion by the temperature gradient on the free surface. The liquid is in contact with an inviscid atmosphere, and the gravity is imposed in the vertical direction, see Fig. 1. Here a horizontal temperature gradient is imposed on the surface of liquid layer and the bottom has zero heat flux. Due to heat transfer, there is also a vertical temperature gradient in the layer. We suppose that the temperature of basic flow $T_{0}$ is linear in $x$ as

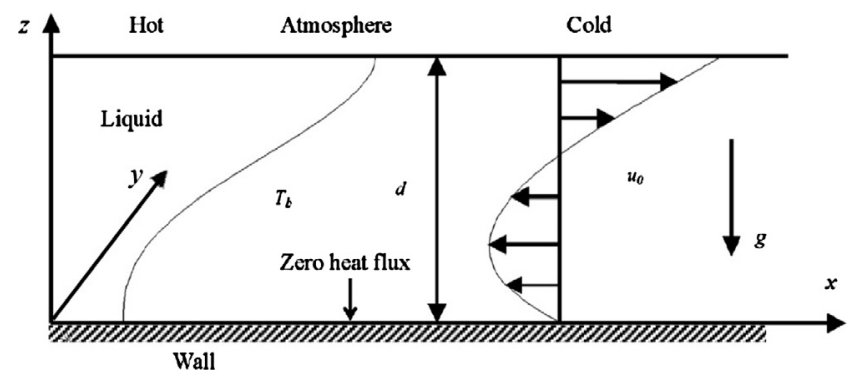

Fig. 1. Schematic of thermocapillary liquid layers in the gravity field. imposed plus a vertical distribution $T_{b}$. The basic flow is assumed to be parallel and $u_{0}$ is the velocity. Here, $d$ is the depth of the layer, $x$ is the streamwise direction, and $z$ is the wall-normal direction. For simplicity, we only consider the case related with Ref. [3], where the surface tension is big enough so that the liquid surface is non-deformable.

This is an approximate model for the convection in a fluid layer with an upper free surface in a long tank, and a temperature difference is maintained between the two endwalls [10]. The relative change of temperature in the liquid layer is assumed to be small. This is true in Ref. [4] for the experiment of silicone oil, where the temperature difference between the two endwalls is less than $10{ }^{\circ} \mathrm{C}$ while the reference temperature of the experiment is about $25^{\circ} \mathrm{C}$. Therefore, the relative changes of density, surface tension, and dynamic viscosity are also small. The variation of density with temperature leads to the buoyancy effect in the presence of gravity, and can be measured by Bond number. The variation of surface tension with temperature leads to the thermocapillary effect and can be measured by Marangoni number. These two dimensionless parameters are considered during the numerical process in the following. However, the variation of dynamic viscosity with temperature is neglected.

\subsection{Governing equations}

In Ref. [10], the surface tension and density of a Newtonian fluid in the gravity field are both assumed to vary linearly with the temperature. The critical parameters of buoyant-thermocapillary flow predicted by linear stability analysis in Ref. [10] are comparable with the experiment [4]. This suggests that the linear relation between the surface tension and temperature is still appropriate for the flow in the presence of gravity. For viscoelastic fluid, it has been observed in the experiments that the surface tension for polymer liquid is also linear with the temperature over limited temperature domains [23]. Therefore, we assume that the surface tension $\tilde{\sigma}$ is related to the temperature $\tilde{T}$ as $\tilde{\sigma}=\tilde{\sigma}_{0}-\gamma\left(\tilde{T}-\tilde{T}_{0}\right)$, where $\gamma$ is the negative rate of change of surface tension with temperature. The Reynolds number $R$ is defined as $R=\frac{\rho U d}{\mu}$, where $\rho, \mu$ are the fluid density, and viscosity, respectively. $U$ is the characteristic velocity defined as $U=b \gamma d / \mu, b$ is the temperature gradient on the surface. The characteristic temperature is $b d$. The Marangoni number is defined as $M a=b \gamma d^{2} / \mu \chi, \chi$ is the thermal diffusivity. There is a relation between $M a$ and $R: M a=R \cdot \operatorname{Pr}$, where $\operatorname{Pr}=\frac{\mu}{\rho \chi}$ is the Prandtl number. The fluid density depends on temperature with the form $\rho=\rho_{0}\left[1-a\left(\tilde{T}-\tilde{T}_{0}\right)\right]$, where $a$ is the thermal expansion coefficient. The gravity effect is measured by the dynamic Bond number $B o=\frac{\rho g a d^{2}}{\gamma}$, and $g$ is the gravitational acceleration. These definitions are the same as those in Ref. [4].

In the presence of gravity, the distribution of fluid density will induce the buoyancy force in the vertical direction. Within Boussinesq's approximation, the dimensionless governing equations are given below [6], which are the continuity equation, the momentum equation and the energy equation, respectively.

$\nabla \cdot \mathbf{u}=0$

$R\left(\frac{\partial \mathbf{u}}{\partial t}+\mathbf{u} \cdot \nabla \mathbf{u}\right)=-\nabla p+\nabla \cdot \mathbf{Q}+B o \cdot T \mathbf{e}_{\mathbf{z}}$

$\frac{\partial T}{\partial t}+\mathbf{u} \cdot \nabla T=\frac{1}{M a} \nabla^{2} T$.

Here $\mathbf{u}, p, T$ are the velocity, pressure and temperature, respectively. For simplicity, the variation of dynamic viscosity with temperature is neglected in (2.2), which is similar to the previous works 
for Newtonian fluid. Generally, the dynamic viscosity of polymer liquid decreases with the temperature and dissipates the perturbation. If we use the viscosity at the lowest temperature in the fluid layer for the characteristic viscosity, then the critical Marangoni number determined in the following is a little higher than the real one.

The constitutive equation for Oldroyd-B fluid [24] is

$\left(1+\lambda \frac{\delta}{\delta t}\right) \mathbf{Q}=\left(1+\lambda \tilde{\beta} \frac{\delta}{\delta t}\right) \mathbf{S}$

$\mathbf{S}$ is the strain-rate tensor,

$\mathbf{S}=\nabla \mathbf{u}+\mathbf{u} \nabla$

$\frac{\delta}{\delta t}$ is the upper convected derivative with the expression of

$\frac{\delta \mathbf{Q}}{\delta t}=\frac{\partial \mathbf{Q}}{\partial t}+\mathbf{u} \cdot \nabla \mathbf{Q}-(\mathbf{u} \nabla) \cdot \mathbf{Q}-\mathbf{Q} \cdot(\nabla \mathbf{u})$.

$\lambda$ is the Weissenberg number, which is defined as $\lambda=\frac{\mu}{\tilde{G}} \frac{U}{d}$, $\tilde{G}$ is the elastic modulus. $\tilde{\beta}$ is the ratio of solvent to the total viscosity [25]. Oldroyd-B fluid recovers Newtonian fluid at $\tilde{\beta}=1$ or $\lambda=0$.

Here we use the stress tensor $\mathbf{Q}$ instead of the extra-stress tensor $\tilde{\mathbf{T}}$ [25] as the former makes the analysis simpler. Indeed, they have the relation $\mathbf{Q}=(1-\tilde{\beta}) \tilde{\mathbf{T}}+\tilde{\beta} \mathbf{S}$. The elastic number $\varepsilon=\lambda / R=\frac{\mu^{2}}{\rho \tilde{G} d^{2}}$ is used to measure the elasticity as it only depends on the properties of the fluid and the flow geometry [25]. Physically, when we only increase the temperature gradient on the free surface $b$, the Weissenberg number and the Reynolds number increase together as they are both proportional to $b$, however, the elastic number is kept constant.

The boundary conditions are set as follows. On the rigid plane, there is no slip and zero heat flux:

$\mathbf{u}=(u, v, w)=0, \quad \frac{\partial T}{\partial z}=0, \quad z=0$,

On the free surface,

$Q_{13}+\frac{\partial T}{\partial x}=0, \quad Q_{23}+\frac{\partial T}{\partial y}=0, \quad w=0$,

$-\frac{\partial T}{\partial z}=B i \cdot\left(T-T_{\infty}\right)+\tilde{Q}, \quad z=1$.

Here, the first two equations stand for the relation between the temperature gradient and stress caused by thermocapillary effect. $Q_{i j}$ is the component of the stress tensor $\mathbf{Q}$, and the subscripts 1 , 2,3 in $Q_{i j}$ stand for the $x, y, z$ directions, respectively. For the basic flow, a horizontal temperature gradient is imposed on the surface in $x$ direction, so $\frac{\partial T}{\partial x}=-1, \frac{\partial T}{\partial y}=0$ at $z=1$. The temperature in basic flow $T_{0}$ is assumed to be

$T_{0}(x, z)=-x+T_{b}(z)$.

When a perturbation appears in the flow, there can be a temperature gradient in both $x$ and $y$ directions. $T_{\infty}$ is the temperature of the bounding gas far from the surface. $B i$ is the Biot number. As the Biot number always makes the flow more stable [3], for simplicity, we set it as zero as those in Refs. [10,22]. $\tilde{Q}$ is the imposed heat flux to the environment, which can be determined by the form of basic flow [3]. In the following solution, $\tilde{Q}=0$.

Here, we consider the case when the liquid layer is an approximate model for the convection in a rectangular slot whose length and width are far larger than its depth $[4,10]$. Therefore, it can be inferred from the conservation of mass that the flow has zero mass flux through any vertical section.

Then the solutions of the basic flow can be derived from the governing equations as follows [6]. $u_{0}(z)=\left(\frac{3}{4} z^{2}-\frac{1}{2} z\right)+B o\left(-\frac{1}{6} z^{3}+\frac{5}{16} z^{2}-\frac{1}{8} z\right), \quad v_{0}=w_{0}=0$

$T_{b}(z)=M a\left[\left(-\frac{1}{16} z^{4}+\frac{1}{12} z^{3}-\frac{1}{48}\right)+B o\left(\frac{1}{120} z^{5}-\frac{5}{192} z^{4}+\frac{1}{48} z^{3}-\frac{1}{320}\right)\right]$

We can see that expression of the basic flow consists of two parts. The first part is the same as the return flow in Ref. [3], while the second part is induced by gravity, and proportional to Bo. The distributions of $u_{0}, T_{b}$ are displayed in Fig. 1. It is observed that the temperature at the bottom is lower than that on the surface, which is opposite to Benard convection and Marangoni convection.

\subsection{Perturbation equations}

Suppose an infinitesimal normal-mode perturbation is added to the basic flow [18],

$(\mathbf{u}, T, P, \mathbf{Q})=\left(\mathbf{u}_{0}, T_{0}, P_{0}, \mathbf{Q}_{0}\right)+(\widehat{\mathbf{u}}, \widehat{T}, \widehat{P}, \widehat{\mathbf{Q}}) \exp [\sigma t+\mathrm{i}(\alpha x+\beta y)]$,

$\mathbf{Q}_{0}=u_{0}^{\prime}(z)\left[\begin{array}{ccc}2 \lambda(1-\tilde{\beta}) u_{0}^{\prime}(z) & 0 & 1 \\ 0 & 0 & 0 \\ 1 & 0 & 0\end{array}\right], \quad \widehat{\mathbf{u}}=(\widehat{u}, \widehat{v}, \widehat{w})$

$\widehat{\mathbf{Q}}=\left[\begin{array}{lll}\hat{Q}_{11} & \hat{Q}_{12} & \hat{Q}_{13} \\ \hat{Q}_{12} & \hat{Q}_{22} & \hat{Q}_{23} \\ \hat{Q}_{13} & \hat{Q}_{23} & \hat{Q}_{33}\end{array}\right]$.

Here the subscript 0 stands for the basic flow and the variables without subscript 0 stand for the perturbation in the following. There is a normal stress in $\mathbf{Q}_{0}$, which is caused by the elasticity of polymer. $\sigma=\sigma_{\mathrm{r}}+\mathrm{i} \sigma_{i}, \sigma_{\mathrm{r}}$ and $\sigma_{i}$ are the growth rate and frequency of small perturbation, respectively, $\alpha, \beta$ denote the wave number in the $x$ and $y$ directions, respectively. In the following, $k=\sqrt{\alpha^{2}+\beta^{2}}$, $\phi=\tan ^{-1}(\beta / \alpha)$, which are the wave number and the direction of wave propagation, respectively. We restrict our attention to the case $\phi \in\left[0^{\circ}, 180^{\circ}\right]$ due to symmetry. This is a little different from the case in Refs. [26-28], where the calculations are performed only for the range $\phi \in\left[0^{\circ}, 90^{\circ}\right]$. As the basic flow of the latter is antisymmetric with respect to the $\mathrm{x}$-axis, the property of the wave propagating in the direction of $\phi$ is the same as that of a wave propagating in the direction of $180^{\circ}-\phi$. So considering the case $\phi \in\left[0^{\circ}, 90^{\circ}\right]$ is enough. However, the waves propagating in the directions of $\phi$ and $180^{\circ}-\phi$ are completely different in this paper. Therefore, we must consider the range $\phi \in\left[0^{\circ}, 180^{\circ}\right]$ in the following, where the case $\phi<90^{\circ}\left(\phi>90^{\circ}\right)$ corresponds to the wave propagating in a direction with a component in the direction of positive (negative) $x$ axis.

Upon linearizing, we can get the equations for perturbation flows by substituting (2.12a) and (2.12b) into the governing equations. The boundary conditions for the perturbation flow are set as follows.

$\widehat{u}=\widehat{v}=\widehat{w}=\frac{\partial \widehat{T}}{\partial z}=0, \quad z=0$,

$\hat{\mathrm{Q}}_{13}+\mathrm{i} \alpha \widehat{T}=0, \hat{\mathrm{Q}}_{23}+\mathrm{i} \beta \widehat{T}=0, \quad \widehat{w}=0, \quad \frac{\partial \widehat{T}}{\partial z}=0, \quad z=1$. 
The perturbation equations are solved by the Chebyshevcollocation method and the details are described in the Appendix A.

\subsection{Code validation}

The code we used is based on our previous work for the problem without gravity [22]. In order to validate our code, we solve the same problems in previous works for Newtonian fluid by setting $\tilde{\beta}=1$. The critical values of thermocapillary-buoyancy convection are displayed in Table 1. The values given by Riley and Neitzel [4] are obtained by experiment while the results of Chan and Chen [10] and present work are derived by linear stability analysis. It can be seen that our results are very close to those of Chan and Chen [10] and the theoretical results compare favorably with the experimental values.

\section{Numerical results}

In the following, we will compute the Marangoni number for the neutral modes $M a_{N}$ and find the critical Marangoni number $M a_{c}$ which is defined as follows

$M a_{c}=\min _{\alpha, \beta} M a_{N}(\operatorname{Pr}, \varepsilon, B o, \tilde{\beta})$.

In order to compare with the case without gravity [22], we restrict our attention to the case for $P r=100$ and $P r=0.02$, $\tilde{\beta}=0.1$. As the property of preferred mode depends on elasticity, the flow stability is examined at different elastic numbers. The results for $\mathrm{Pr}=100$ and $\mathrm{Pr}=0.02$ are presented in Sections 3.1 and 3.2, respectively. The perturbation field is displayed in Section 3.3 while the energy mechanism is analyzed in Section 3.4.

\section{1. $\operatorname{Pr}=100$}

The variation of critical Marangoni number $M a_{c}$ with $B o$ at $\operatorname{Pr}=100, \varepsilon \leqslant 0.1$ is displayed in Fig. 2. For Newtonian fluid $(\varepsilon=0), M a_{c}$ increases with $B o$ and the preferred mode changes from oblique wave $\left(\phi \neq 0^{\circ}, 90^{\circ}\right)$ to streamwise wave $\left(\phi=0^{\circ}\right)$ when $B o>0.1$. This trend is the same as that reported for $\operatorname{Pr}=13.9$ [10]. For $\varepsilon=0.05, M a_{c}$ still increases with $B o$. The preferred mode changes from oblique wave to streamwise wave when $B o>0.184$ and changes to oblique wave again when $B o>0.823$. For $\varepsilon=0.1, M a_{c}$ increases with $B o$ at first and the variation of preferred mode is similar to the case for $\varepsilon=0.05$. However, when $B o>2.064, M a_{c}$ decreases with $B o$ and the preferred mode changes to the spanwise stationary mode $\left(\phi=90^{\circ}, \sigma_{i}=0\right)$.

Generally, when Bo increases, the preferred mode changes from oblique wave to streamwise wave and back to oblique wave. Many different kinds of modes are excited. For example the streamwise

\section{Table 1}

The critical parameters for the hydrothermal waves at $\operatorname{Pr}=13.9, B o=0.142$. Comparison with reference values by experiment. The definitions of Marangoni number and angle of propagation are the same as those in the work of Chan and Chen [10].

\begin{tabular}{llll}
\hline & $\begin{array}{l}\text { Experiment } \\
\text { Riley and } \\
\text { Neitzel }\end{array}$ & \multicolumn{2}{l}{ Linear stability analysis } \\
\cline { 3 - 4 } & 26.91 & $\begin{array}{l}\text { Chan and } \\
\text { Chen }\end{array}$ & $\begin{array}{l}\text { Present } \\
\text { work }\end{array}$ \\
\hline$M a$ & $23.2^{\circ}$ & 24.70 & 24.72 \\
Angle of propagation & 2.58 & $21.7^{\circ}$ & $21.9^{\circ}$ \\
Wavelength & 0.0217 & 2.48 & 2.47 \\
Dimensionless frequency & 0.0237 & 0.0234 \\
Dimensionless phase & 0.0561 & 0.0583 & 0.0579 \\
speed & & & \\
\hline
\end{tabular}

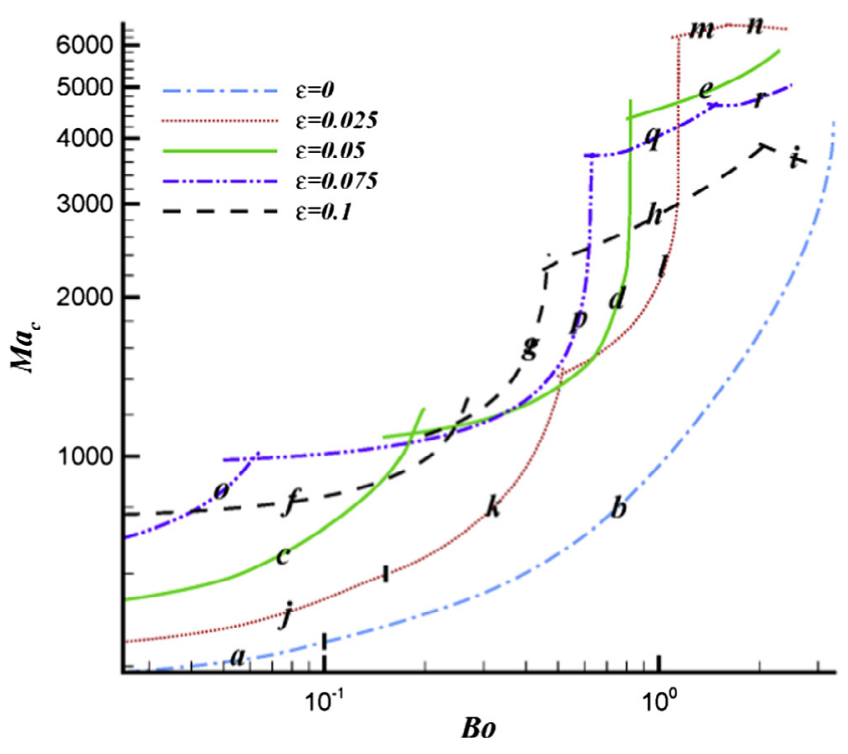

Fig. 2. The variation of $M a_{c}$ with $B o$ at $\operatorname{Pr}=100, \varepsilon \leqslant 0.1$. The modes at different elastic numbers are (1) $\varepsilon=0$ : (a) oblique wave, (b) streamwise wave; (2) $\varepsilon=0.025$ : (j) oblique wave, (k) streamwise wave, (l) streamwise wave, (m) oblique wave, (n) oblique wave; (3) $\varepsilon=0.05$ : (c) oblique wave, (d) streamwise wave, (e) oblique wave; (4) $\varepsilon=0.075$ : (o) oblique wave, (p) streamwise wave; (q) oblique wave, (r) oblique wave; (5) $\varepsilon=0.1$ : (f) oblique wave, (g) streamwise wave, (h) oblique wave, (i) spanwise stationary mode.

wave, which is not found for $P r=100$ in the absence of gravity [22]. This can only be due to the effect of gravity. Additionally, there are two different kinds of streamwise wave for $\varepsilon=0.025$ while both the upstream and downstream oblique waves are found for $\varepsilon=0.025-0.1$. The spanwise stationary mode is also excited by gravity while it can only be the preferred mode at $\varepsilon>0.3$ in the flow without gravity [22].

The wave number corresponding to the mode in Fig. 2 is displayed in Fig. 3. Although the variation of preferred mode for different $\varepsilon$ is similar, the variation of the corresponding wave number differs from each other. For streamwise wave, the wave numbers for (b), (l) increase with $B o$, whereas the reverse is the case for $(\mathrm{d}),(\mathrm{p})$ and $(\mathrm{g})$. The variation for $(\mathrm{k})$ is not monotonous.

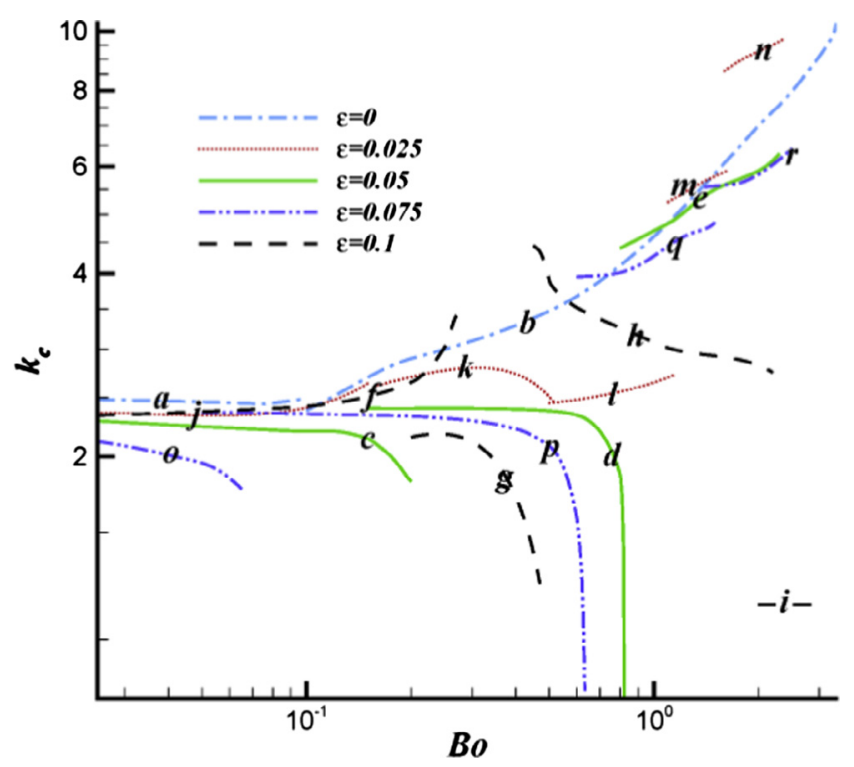

Fig. 3. The wave number corresponding to the mode in Fig. 2. 
For oblique wave, the wave numbers for (c), (h) and (o) decrease gradually with $B o$ while the opposite happens for (e), (f), (m), (n), (q) and (r). The wave number for (a) has little change. Meanwhile, the corresponding wave number for (i) (spanwise stationary mode) is larger than zero. In contrast, the wave number of spanwise stationary mode tends to zero in the flow without gravity [22].

The wave propagation angle corresponding to the oblique wave in Fig. 2 is displayed in Fig. 4. It can be found that the oblique waves for (a), (c), (j), (o) and (n) are upstream while those for (e), $(\mathrm{f}),(\mathrm{m})$ are downstream. The waves (q) and (r) change from upstream to downstream while the mode $(\mathrm{h})$ is nearly spanwise.

The wave speed $C=\left|\sigma_{i}\right| / k$ corresponding to the mode in Fig. 2 is displayed in Fig. 5. Most of the wave speeds have gentle variations. It can be seen that the wave speeds of (a), (j), (c), (o) are nearly the same when $B o \leqslant 0.1$, although their critical Marangoni numbers differ from each other. For the spanwise stationary mode (i), its wave speed is zero.

The variation of $M a_{c}$ with $B o$ at $\operatorname{Pr}=100, \varepsilon=0.3,0.4$ is displayed in Fig. 6. It can be seen that $M a_{c}$ always increases with $B o$. The preferred mode consists of spanwise stationary mode (a), (c), (d), (e) and oblique wave (b). However, the corresponding wave numbers for curves (a) and (d) tend to zero, which is the same as the case without gravity [22], while those for curve (c) and (e) are obviously larger than zero (see Fig. 7).

The propagation angle and wave speed corresponding to the oblique wave in Fig. 6 are displayed in Figs. 8 and 9, respectively. When $B o<2$, there is a slight reduction for the propagation angle. After that, the propagation angle tends to $90^{\circ}$ while the wave speed tends to zero, which means that the oblique wave tends to the spanwise stationary mode. The variation of wave speed with Bo is just opposite to that of the propagation angle. For the spanwise stationary mode (a), (c), (d), (e), the propagation angle is $90^{\circ}$ while the wave speed is zero.

\section{2. $\operatorname{Pr}=0.02$}

The variation of $M a_{c}$ with $B o$ at $P r=0.02$ is displayed in Fig. 10 . It can be seen that $M a_{c}$ always decreases with $B o$. The preferred mode for Newtonian fluid $(\varepsilon=0)$ is the oblique wave. When Bo increases, the propagation angle decreases with $B o$, and the wave changes from upstream to downstream. This trend is similar to that reported for small Prandtl numbers [6]. For $\varepsilon=0.012$, it

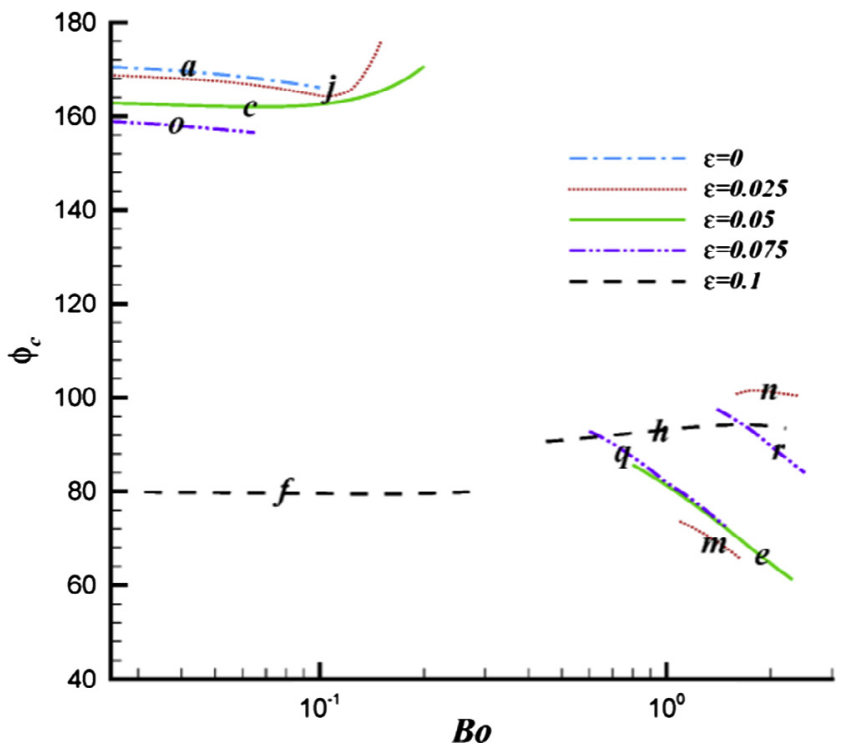

Fig. 4. The wave propagation angle corresponding to the mode in Fig. 2.

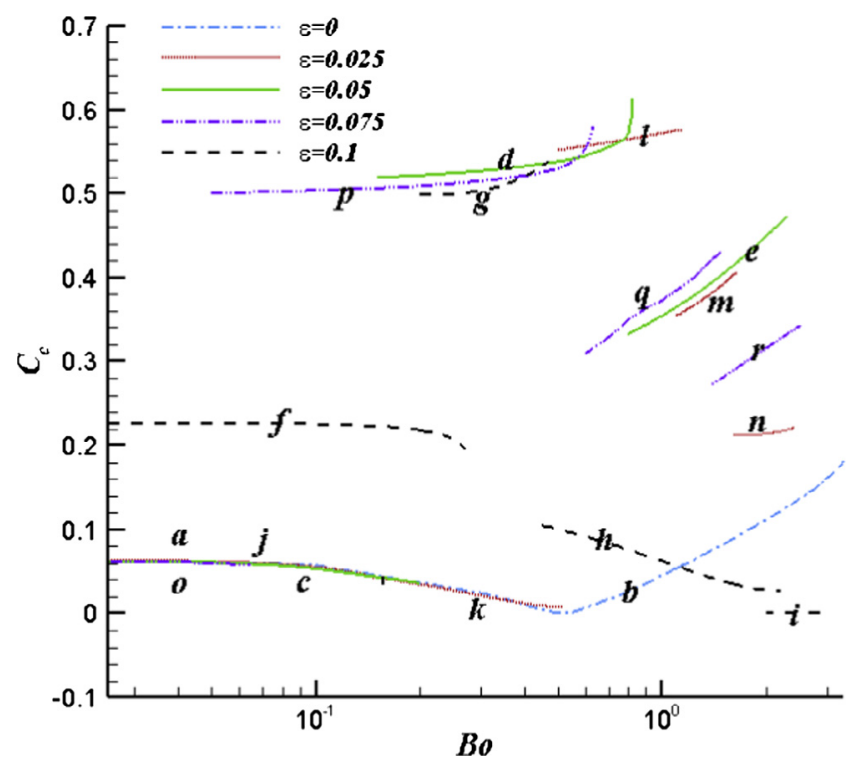

Fig. 5. The wave speed corresponding to the mode in Fig. 2.

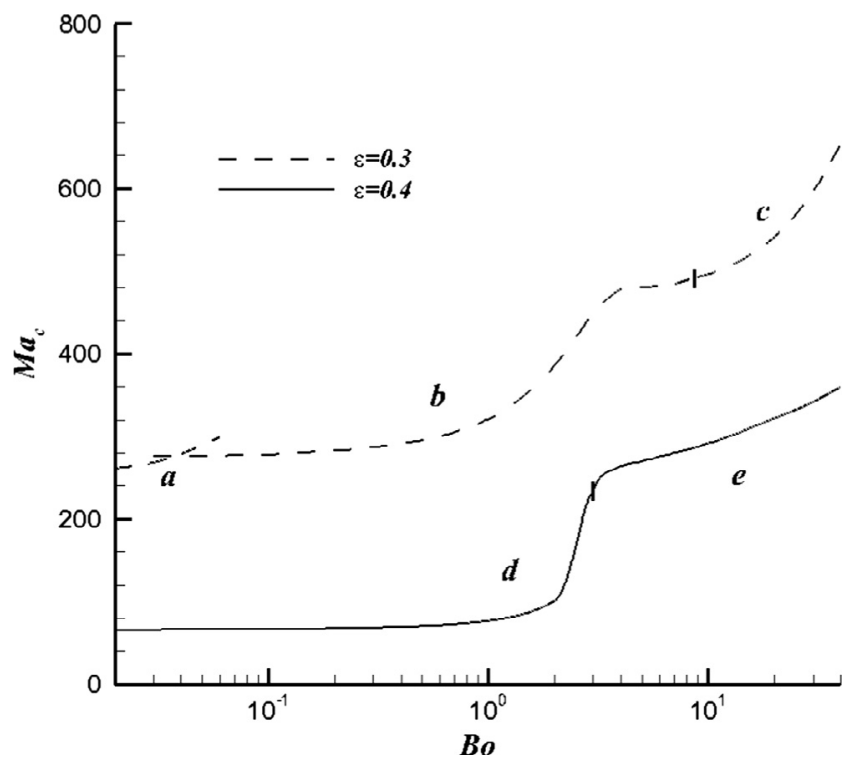

Fig. 6. The variation of $M a_{c}$ with $B o$ at $\operatorname{Pr}=100, \varepsilon=0.3,0.4$. The modes at different elastic numbers are (1) $\varepsilon=0.3$ : (a) spanwise stationary mode, (b) oblique wave, (c) spanwise stationary mode; (2) $\varepsilon=0.4$ : (d) spanwise stationary mode, (e) spanwise stationary mode.

changes to the streamwise wave. For $\varepsilon=0.03$, the preferred mode changes from spanwise stationary mode to streamwise wave when Bo $>2.76$. For $\varepsilon=0.07$, the preferred mode is always the spanwise stationary mode.

The wave number and wave speed corresponding to the mode in Fig. 10 are displayed in Figs. 11 and 12, respectively. It is observed that the wave numbers have gentle variations for most of the modes. Especially for (a), the wave number nearly keeps the same although the corresponding critical Marangoni number decreases a lot. The variations of wave number for the streamwise wave (b), (e) and (c) are opposite. For (a), (b), (c), (e), the wave speed increases with Bo obviously.

For spanwise stationary mode (d) and (f), the wave speed is zero, and the wave number tends to zero, which is the same as that in the flow without gravity [22]. It seems that for $\operatorname{Pr}=0.02$, the 


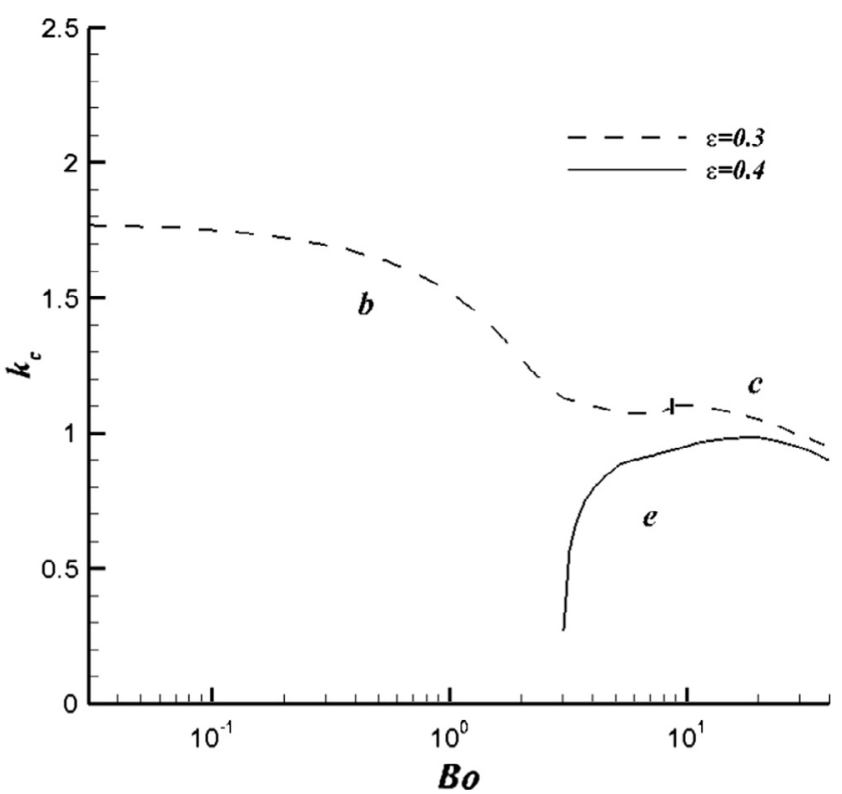

Fig. 7. The wave number corresponding to the mode in Fig. 6.

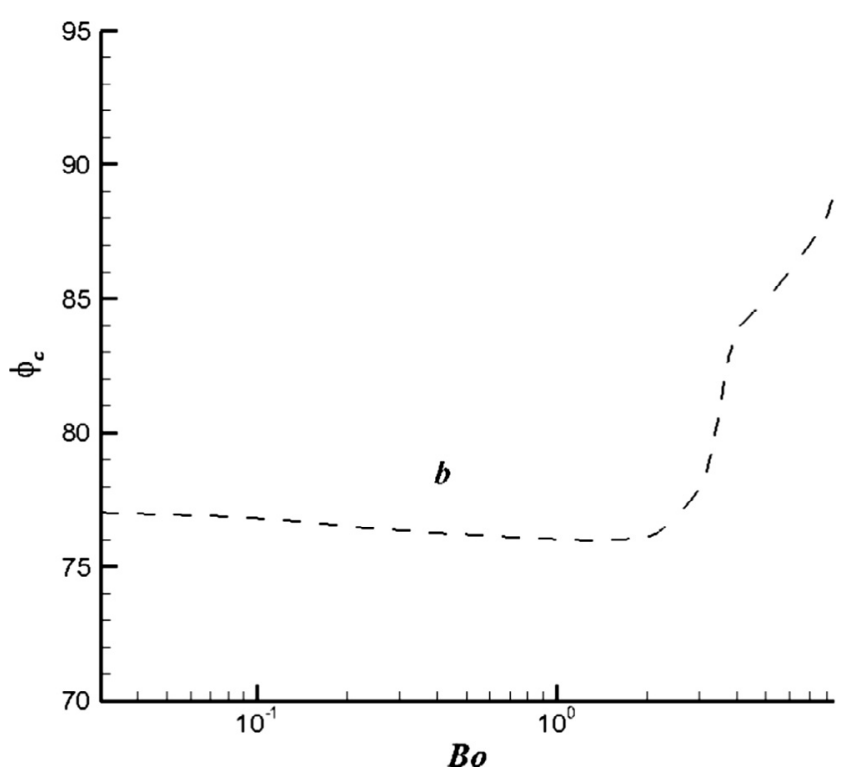

Fig. 8. The wave propagation angle corresponding to the oblique wave in Fig. 6 .

gravity cannot excite the spanwise stationary mode with $k_{c}>0$ as that that for $\operatorname{Pr}=100$.

\subsection{The perturbation flow field}

The perturbation flow field is studied in this section. It is found that the influence of gravity for perturbation mode mainly appears in the spanwise stationary mode at $P r=100$. In Fig. 13, the streamlines and isothermals are plotted in the direction of the wave vector. We can find the rolls which are periodically arranged in the perturbation velocity field. However, comparing with those in the flow without gravity [22], the cores of rolls are closer to the free surface. The temperature fluctuates in the vertical direction. This is in contrast to the former case where the isothermals for spanwise stationary modes are all vertical lines.

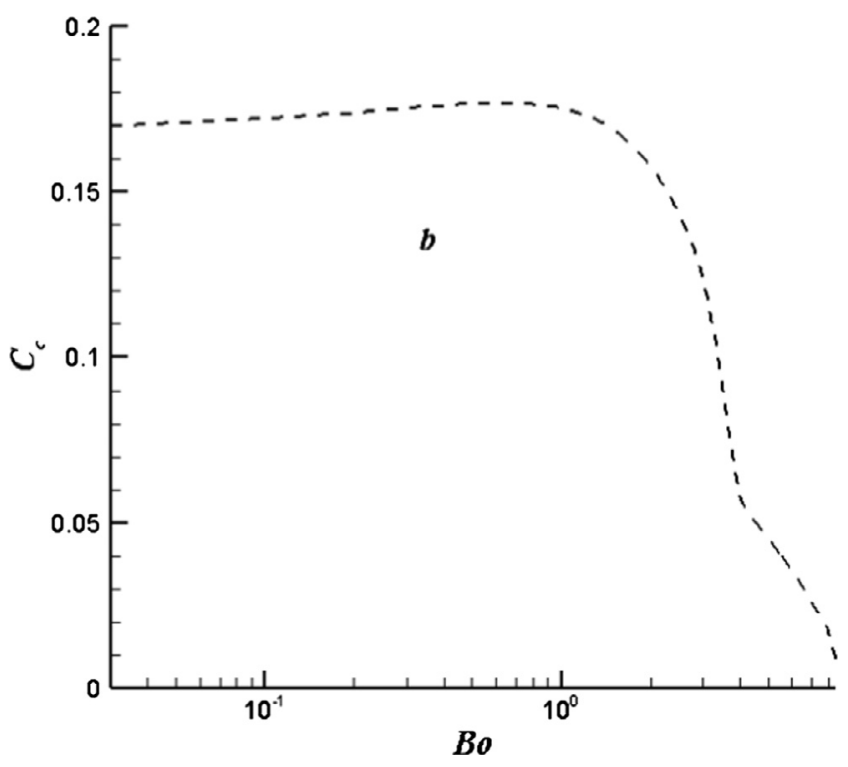

Fig. 9. The wave speed corresponding to the oblique wave in Fig. 6.

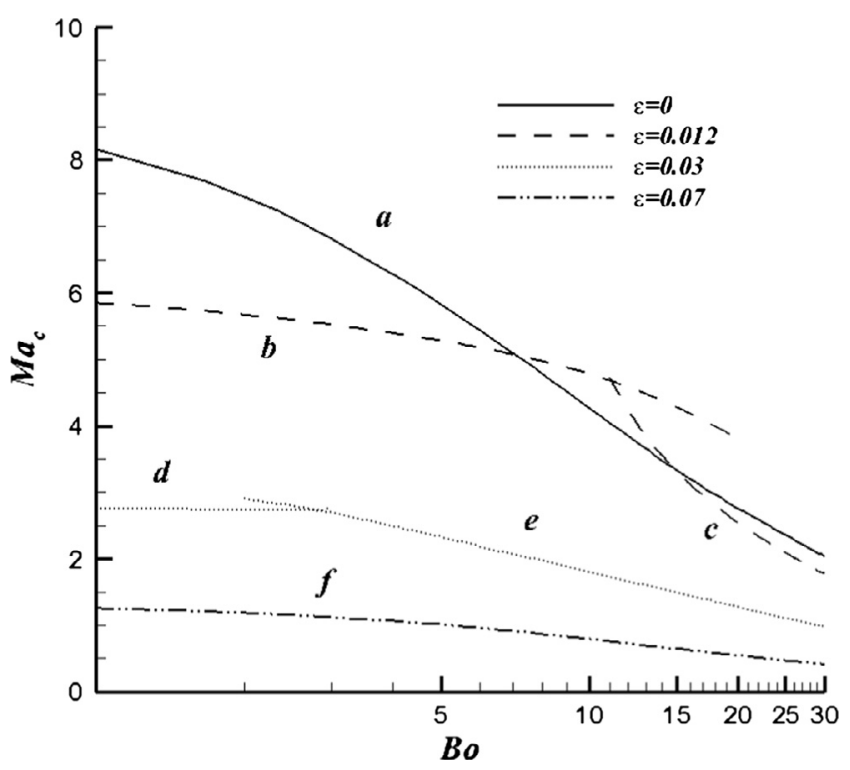

Fig. 10. The variation of $M a_{c}$ with $B o$ at $P r=0.02, \varepsilon \leqslant 0.07$. The modes at different elastic numbers are (1) $\varepsilon=0$ : (a) oblique wave; (2) $\varepsilon=0.012$ : (b) streamwise wave, (c) streamwise wave; (3) $\varepsilon=0.03$ : (d) spanwise stationary mode, (e) streamwise wave; (4) $\varepsilon=0.07$ : (f) spanwise stationary mode.

The mechanism can be explained as follows. We pay attention to the temperature distribution in the vertical direction. The gradient of vertical temperature for basic flow increases with Bo. As the heat convection is more significant than the heat conduction for $\operatorname{Pr}=100$, the convective cooling $\left|\widehat{W} \frac{\partial T_{0}}{\partial z}\right|$ below the hot spot on the surface also increases. However, the vertical velocity $\widehat{w}$ is small near the upper and lower boundaries, the cooling effect reaches a maximum in the middle region $(z \approx 0.7)$, then a cold spot appears. In contrast, this change does not happen at $P r=0.02$. The reason is that the relative importance of heat convection and heat conduction at $P r=0.02$ is opposite to the case at $P r=100$. Thus the convective cooling in vertical direction has little influence on the temperature distribution. 


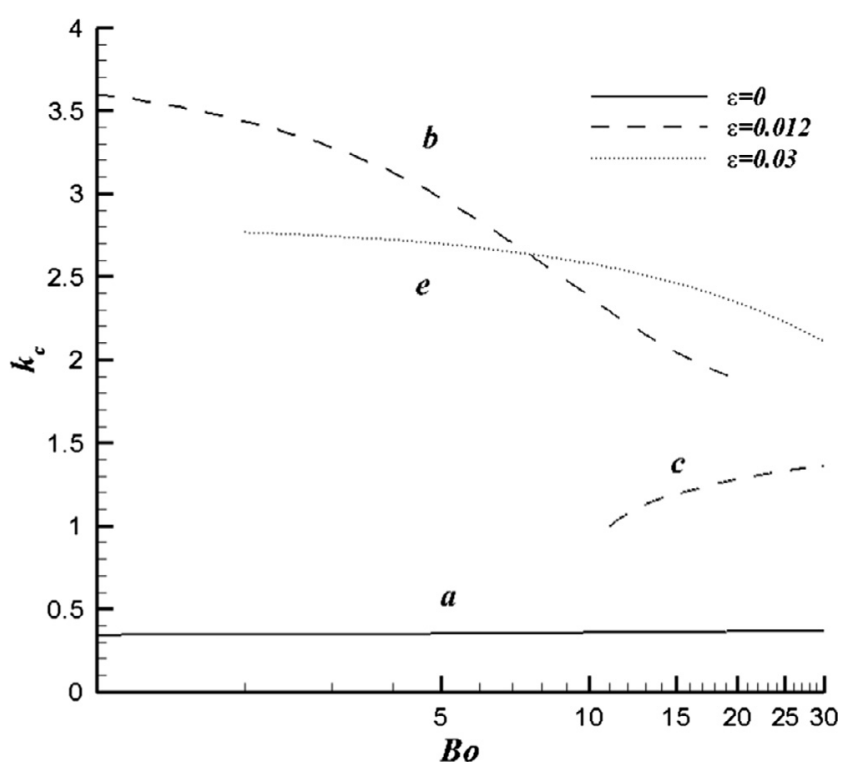

Fig. 11. The wave number corresponding to the mode in Fig. 10.

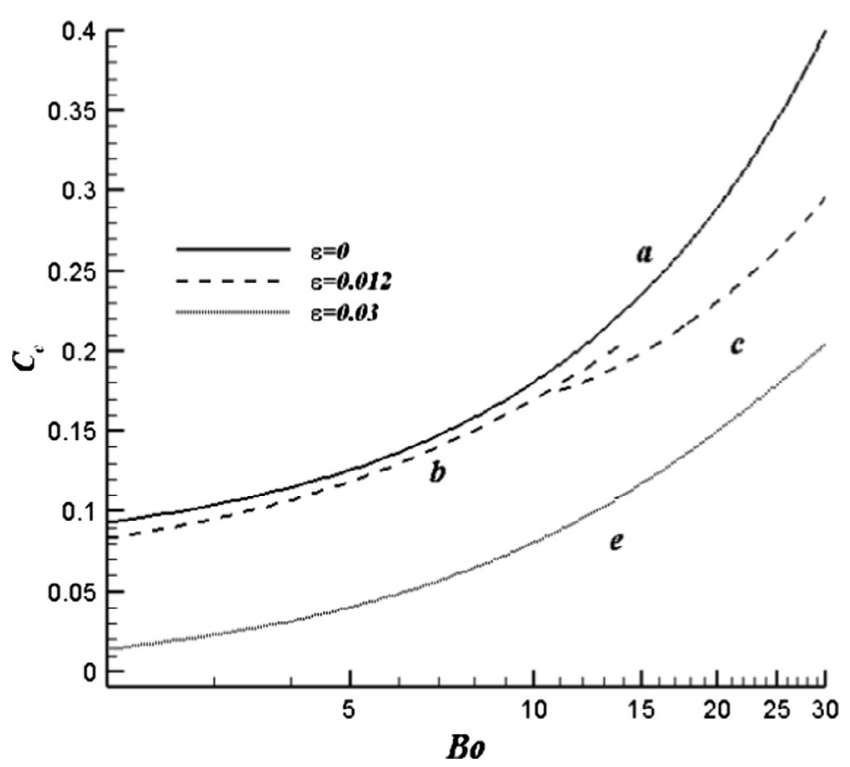

Fig. 12. The wave speed corresponding to the mode in Fig. 10.
Another obvious change of perturbation field is that the gravity excites some preferred modes with high wave numbers $O\left(k_{c}\right)=10$ (Fig. 3). Although the increasing of wave number often leads to high dissipation, the relative scale of vertical velocity also increases, which adds the vertical heat convection at $\mathrm{Pr}=100$. Then the temperature fluctuation is enhanced and the temperature gradient on the surface increases. Thus, the work done by the Marangoni force can counteract the dissipation. This effect is more prominent when $B o$ increases, as the gravity increases the gradient of vertical temperature for basic flow as well as the vertical heat convection.

\subsection{Energy analysis}

Then we study the energy mechanism in this section. The rate of change for perturbation energy can be derived as follows [29-31]

$$
\begin{aligned}
\frac{\partial E_{k i n}}{\partial t}= & -\frac{1}{2 R} \int(\mathbf{Q}: \mathbf{S}) \mathrm{d}^{3} r+\frac{1}{R} \int \mathbf{u} \cdot \mathbf{Q} \cdot \mathbf{n} \mathrm{d}^{2} r \\
& -\int \mathbf{u} \cdot\left((\mathbf{u} \cdot \nabla) \mathbf{u}_{0}\right) \mathrm{d}^{3} r+\int\left(\frac{B o}{R} T \mathbf{e}_{\mathbf{z}} \cdot \mathbf{u}\right) \mathrm{d}^{3} r \\
& =-N+M+I+G,
\end{aligned}
$$

where $N$ is the work done by the perturbation stress, $M$ is the work done by Marangoni forces on the free surface, $I$ is the interaction between the perturbation flow and the basic flow, $G$ is the work done by gravity, respectively.

We also consider the cases when Bo is large enough to show the gravity effect. Table 2 shows the four terms in (3.2) for streamwise and oblique waves. Here, the perturbation is normalized as follows:

$\int \mathbf{u}^{2} \mathrm{~d}^{3} r=1$

For $\operatorname{Pr}=100, G$ is always negative for streamwise and oblique waves, which means that the gravity does negative work for these two modes. Comparing with the case without gravity ( $N, M$ are both positive, $I$ is negligible), although $N$ and $M$ are still the major terms, $I$ and $G$ are not negligible anymore. The growing importance of $I$ is due the gravity as $u_{0}^{\prime}$ increases with $B o$.

For $\operatorname{Pr}=0.02, G$ is always positive for oblique wave and can even be larger than $M$ and $I$ when $B o=20$, which suggests that the energy mechanism has changed by the gravity significantly. This can be explained from (3.2). As there is no obvious change for the shape of oblique wave when $B o$ increases, the relative importance of $G$ and $N$ is approximately proportional to $B o$. Therefore, $G$ can be the most important energy source for the

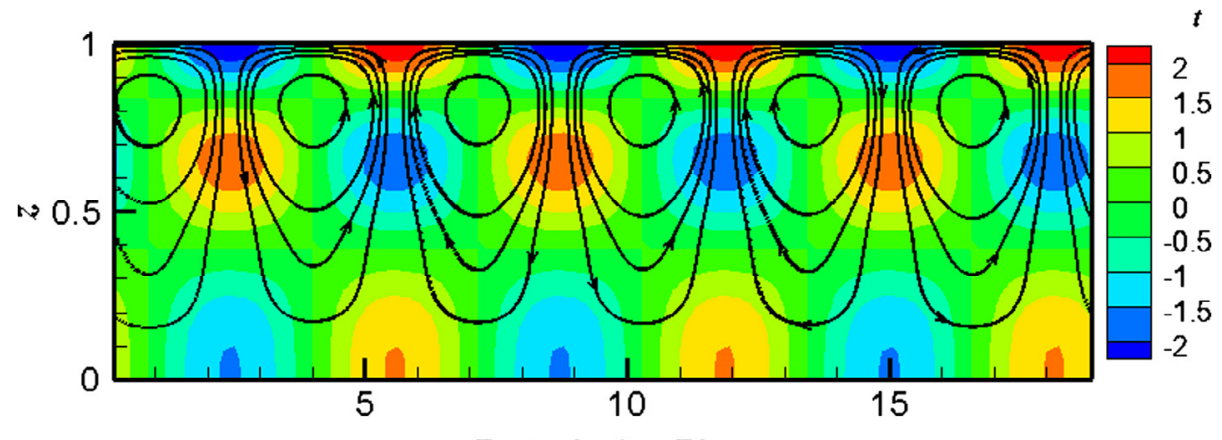

Perturbation Phase

Fig. 13. The perturbation flow field of the spanwise stationary mode at $\operatorname{Pr}=100, B o=20, \varepsilon=0.4, M a=321.3, k=0.98$. 
Table 2

The terms of perturbation energy growth for the streamwise and oblique waves. Here, SW stands for streamwise wave while OW stands for oblique wave.

\begin{tabular}{|c|c|c|c|c|}
\hline & $-N$ & $M$ & $I$ & $G$ \\
\hline \multicolumn{5}{|l|}{$\operatorname{Pr}=100$} \\
\hline SW: $\varepsilon=0, B o=2, M a=1707$ & -25.195293 & 27.542466 & 0.004167 & -2.351477 \\
\hline OW: $\varepsilon=0.05, B o=1.5, M a=4992$ & -0.526221 & 0.476968 & 0.052677 & -0.003435 \\
\hline OW: $\varepsilon=0.1, B o=1, M a=2863$ & -0.058735 & 0.078221 & -0.011770 & -0.006863 \\
\hline OW: $\varepsilon=0.3, B o=8, M a=488.6$ & -0.748298 & 0.947723 & -0.033867 & -0.165494 \\
\hline \multicolumn{5}{|l|}{$\operatorname{Pr}=0.02$} \\
\hline $\mathrm{OW}: \varepsilon=0, B o=3, M a=6.82$ & -0.036665 & 0.024213 & 0.006566 & 0.005898 \\
\hline OW: $\varepsilon=0.005, B o=20, M a=3.15$ & -0.076925 & 0.016115 & 0.022756 & 0.038123 \\
\hline $\mathrm{SW}: \varepsilon=0.012, B o=20, M a=2.54$ & -0.113164 & -0.004795 & 0.128285 & -0.010239 \\
\hline SW: $\varepsilon=0.03, B o=5, M a=2.33$ & 0.023140 & -0.001677 & -0.020824 & -0.000374 \\
\hline
\end{tabular}

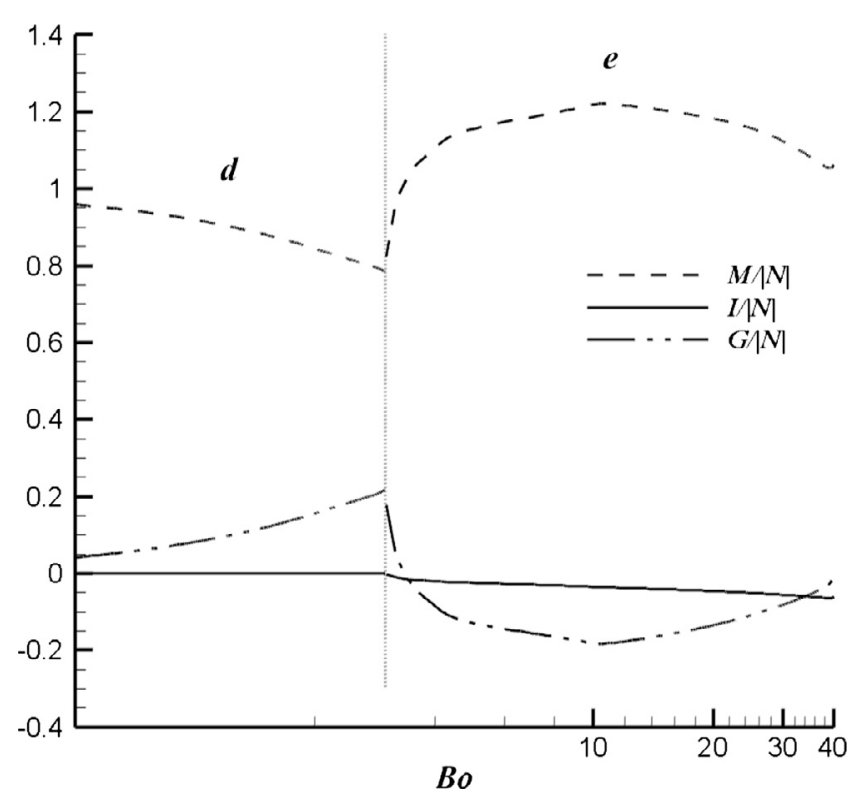

Fig. 14. The ratios of four terms in (3.2) for the spanwise stationary mode at $\operatorname{Pr}=100, \varepsilon=0.4$.

perturbation when $B o$ is large enough. However, for streamwise wave, $N, M, I$ can still change sign in different parameters, just as the case without gravity [22]. This suggests that its energy mechanism mainly depends on elasticity.

For spanwise stationary mode, we plot the ratios of four terms in Figs. 14 and 15. In these cases, $N$ is always positive, which means that the perturbation stress dissipates the energy. For $P r=100, I$ is negligible in most cases. $G$ can be positive in the mode of (d) and (e), which contrasts to the other cases at $P r=100 . G / N$ increases with $B o$ for (d) while its variation for (e) is not monotonous. However, even when the gravity does positive work for the perturbation, $M a_{c}$ still keeps increasing with Bo in Fig. 6. For $\operatorname{Pr}=0.02, I$ is also negligible. $M$ and $G$ are the main energy source. $G / N$ keeps increasing with $B o$ and exceeds $50 \%$ when $B o>9$.

\section{Discussion}

In this section, we will discuss the effect of gravity on the instability mechanism and the properties of preferred modes.

The gravity can influence the flow stability in two ways. First, the basic flow which appears in the perturbation equation depends on the gravity. The eigenvalue is determined as a function of $B$. The increasing of $B o$ leads to the variation of $M a_{c}$. The type of preferred mode can also be changed at some critical value of $\mathrm{Bo}$. Second, the energy analysis shows that the gravity can do work for the

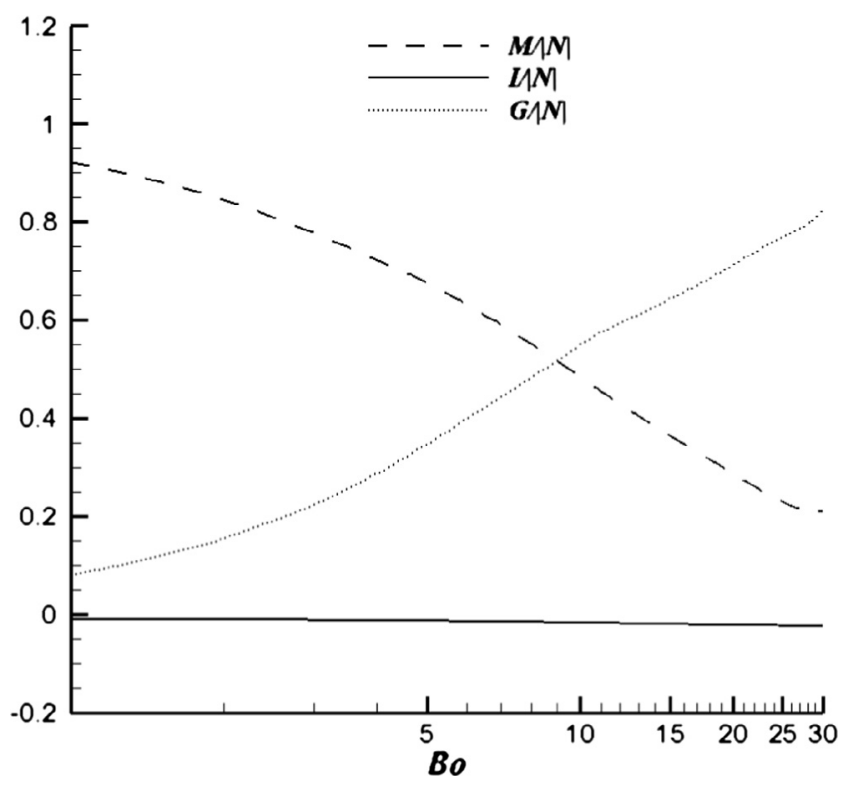

Fig. 15. The ratios of four terms in (3.2) for the spanwise stationary mode at $\operatorname{Pr}=0.02, \varepsilon=0.07$.

perturbation. The work can either be positive or negative, which depends on the mode and parameters. These two effects change the instability mechanism significantly. The cases for three kinds of modes are discussed respectively in the following.

\section{(1) Spanwise stationary mode}

For spanwise stationary mode, the energy analysis shows that the major terms $N, M$ are both positive, while $I$ is negligible. Thus, the thermocapillary force is the driving force while the perturbation stress causes damping.

For $\operatorname{Pr}=100, M a_{c}$ increases with $B o$ when $B o$ is small. This can be explained in the view of temperature. In these cases, $\left|\widehat{u} \frac{\partial T_{0}}{\partial x}\right|,\left|\widehat{w} \frac{\partial T_{0}}{\partial z}\right|$ have the same order of magnitude, which means the horizontal convection and vertical convection are both important in the temperature field. The vertical velocity $w>0$ when underneath the hot spot on the surface, which produces a vertical convective cooling. When Bo increases, the gradient of vertical temperature for basic flow $\frac{\partial T_{0}}{\partial z}$ becomes larger. The convective cooling $\left|\widehat{W} \frac{\partial T_{0}}{\partial z}\right|$ also increases, which leads to the decreasing of the temperature for the hot spot. Thus, the flow becomes more stable, and $M a_{c}$ increases with $B o$.

However, when Bo becomes larger, the effect of gravity is deeply coupled with elasticity and thermocapillary force. The critical wave number for spanwise stationary mode becomes larger than 
zero, and the flow field is also very different from the case when $B o$ is small. $M a_{c}$ can decrease with Bo for the curve (i) in Fig. 2. Meanwhile, the work done by gravity is not directly related to the variation of $M a_{c}$ with Bo. $G$ can be positive when $M a_{c}$ increases with $B o$ (curve (a),(d) in Fig. 6) while $G$ can be negative when $M a_{c}$ decreases with Bo (curve (i) in Fig. 2).

For $\operatorname{Pr}=0.02$, the case is similar to that without gravity. The computation shows that $\left|\widehat{u} \frac{\partial T_{0}}{\partial x}\right| \gg\left|\widehat{w} \frac{\partial T_{0}}{\partial z}\right|$ for $z>0$. The horizontal convection is dominant in the temperature field. Then, the variation of $\frac{\partial T_{0}}{\partial z}$ with $B o$ has little effect on the temperature distribution. The streamwise velocity $u>0$ when underneath the hot spot on the surface, which produces a horizontal convective heating. As the point moving towards the hot spot on the surface has a higher temperature than that on the surface, the gravity does positive work for this convection. $M a_{c}$ also decreases with $B$.

The spanwise stationary mode does not appear in return flow for Newtonian fluid. The vertical temperature distribution in return flow corresponds to the layer being cooled from below, which is in contrast to the classical Marangoni instability for the layer being heated from below [3]. The presence of gravity even increases the vertical temperature gradient. Therefore, the stationary instability cannot be found for Newtonian fluid. However, for viscoelastic fluid, due to the normal stress in basic flow, the vertical convection can lead to a stress in the streamwise direction. It makes a streamwise flow, which produce a convective heating and destabilize the flow. This is the reason why spanwise stationary mode appears in Oldroyd-B fluid.

\section{(2) Oblique wave}

For the oblique waves at $\operatorname{Pr}=100, \varepsilon \leqslant 0.1$, the vertical convection is dominant for the temperature field. The waves can be divided into two types: curve (a), (c), (j), (o), and curve (e), (f), (h), (m), (n), (n) (r) in Fig. 2. The former is close to streamwise. The phase difference between the perturbation temperature and the vertical velocity is nearly $180^{\circ}$, and gravity does negative work for the perturbation. The latter is close to spanwise. Its corresponding phase difference is nearly $90^{\circ}$, and the work done by gravity is almost zero.

For $\operatorname{Pr}=100, \varepsilon=0.3$ (curve (b) in Fig. 6), the corresponding phase difference is larger than $90^{\circ}$ and $G$ is negative, while for $\operatorname{Pr}=0.02, \varepsilon=0$ (curve (a) in Fig. 10), the corresponding phase difference is close to zero and $G$ is positive. However, both of them are nearly spanwise and their frequencies are small. In some extent, their mechanisms are similar to those for spanwise stationary mode.

For Newtonian fluid, the gravity does not change the instability of oblique wave qualitatively (see Figs. 2 and 10). However, the case for Oldroyd-B fluid is more complicated. When both gravity and elasticity are small, the elasticity leads to a phase difference between the Marangoni force and velocity on the free surface, which makes a decreasing of the work done by Marangoni force. The flow is stabilized by the elasticity. When the gravity is deeply coupled to elasticity, more kinds of oblique waves are excited (see Figs. 2 and 6).

\section{(3) Streamwise wave}

The streamwise wave is not the preferred mode for $P r=100$ in the flow without gravity [22]. In contrast, it becomes the preferred mode at $\operatorname{Pr}=100, \varepsilon \leqslant 0.1$ (curve (b),(d),(g), (k), (l), (p) in Fig. 2) in this work. They can also be divided into two types. The properties of Curve (b) and (k) are similar to those for the oblique waves (curve (a), (c) in Fig. 2), while for Curve (d), (g), (l) and (p), the corresponding phase difference is nearly $90^{\circ}$, which makes $G$ almost zero.
For the streamwise wave at $P r=0.02$ (curve (b), (c),(e) in Fig. 10), the energy analysis shows that all of $N, M, I$ can change sign in different parameters, which is the same as the case in the flow without gravity [22]. Comparing with $N$ and $I, M$ and $G$ are not the main terms. The elasticity is the most important factor for the instability mechanism. The streamwise velocity fluctuates several times in the vertical direction, which is distinct from all of the previous modes.

Although streamwise wave appears in both Newtonian fluid and Oldroyd-B fluid, there are many differences between them. For $P r=100$, the wave number of streamwise wave for Newtonian fluid is increased by gravity significantly when $B o$ is order 1 , which is opposite to the case for Oldroyd-B fluid. For $P r=0.02$, there is no streamwise wave for Newtonian fluid, while the elasticity excites the streamwise wave with vertical fluctuations for Oldroyd-B.

Then, we summarize the variation of critical Marangoni number and preferred mode with gravity. $M a_{c}$ increases with $B o$ for $\operatorname{Pr}=100$ except the curve (i) in Fig. 2 while it always decreases with $B o$ for $\operatorname{Pr}=0.02$. The variation of $M a_{c}$ is very obvious when $O(B o)=1$ for $\operatorname{Pr}=100, \varepsilon \leqslant 0.1$, while in other cases we studied, the corresponding Bond number has $O(B o)=10$. There may be two reasons for this difference. First, we confine ourselves to the governing equation of temperature perturbation. When $\operatorname{Pr}=100$, the heat convection is more significant than the heat conduction for the temperature variation, while the case for $\operatorname{Pr}=0.02$ is the opposite. Thus, the variation of vertical convection with $B o$ will have a more important influence on the temperature field for the former than for the latter, and the eigenvalue for the former is more sensitive to the change of Bo. Second, as the instability mechanism is deeply affected by elasticity for $\operatorname{Pr}=100, \varepsilon=0.3,0.7$, the effect of gravity is more obvious when $\varepsilon \leqslant 0.1$.

The type of preferred mode changes several times for $\operatorname{Pr}=100$, especially when $\varepsilon \leqslant 0.1$. However, there are fewer changes for $\operatorname{Pr}=0.02$. This may be due to the increasing of $M a_{c}$ for the former. The Weissenberg number $\lambda$ of the preferred mode also increases a lot with $B$ o for $P r=100$. Therefore, there are remarkable growths for the gravity, elasticity and thermocapillary forces together. These forces are deeply coupled and more kinds of modes are excited in the flow. As a result, the opportunity for the change of preferred mode also increases. In contrast, $M a_{c}$ and $\lambda$ decrease with $B o$ for the latter, and the properties of its preferred modes have little changes.

Comparing with the case without gravity [22], the presence of gravity not only changes the critical Marangoni number, but also makes the instability mechanism more complex. Besides three kinds of modes in Ref. [22], the coupling of gravity to elasticity and thermocapillary force excites more kinds of preferred modes for $\operatorname{Pr}=100$, especially the streamwise wave and the spanwise stationary mode with $k>0$, which cannot be found in the absence of gravity [22]. Meanwhile, the work done by gravity becomes a new energy source for perturbation. It can even be the most important source for $\operatorname{Pr}=0.02$ when $B o$ is large enough (see Table 2 and Fig. 15) although there is little change for its mode property. The gravity also increases the importance of $I$ in the energy mechanism at $\operatorname{Pr}=100$. Finally, the gravity increases the gradient of vertical temperature for basic flow. The temperature distribution of spanwise stationary mode is highly affected by the vertical convective cooling for $\operatorname{Pr}=100$ (see Fig. 13), and some modes with high wave numbers are excited.

Although the variation of critical Marangoni number with gravity in this work seems similar to that in the buoyantthermocapillary convection of Newtonian fluid, the instability mechanism here strongly depends on the elasticity. The appearance of spanwise stationary mode in return flow and the streamwise wave with vertical fluctuation can only be attributed to the elasticity, as they are not found in Newtonian fluid. For small 
elastic numbers, the change of preferred mode occurs more frequently than the case of Newtonian fluid [10] (see Figs. 2 and 3). When the elastic number is large enough, the preferred mode becomes the spanwise stationary mode. $M a_{c}$ decreases with $\varepsilon$ significantly $\left(O\left(M a_{c}\right)=100, O(R)=1\right.$ for $\varepsilon=0.4$ in Fig. 6). Therefore, we cannot find the preferred mode with $O\left(k_{c}\right)=10$ as the case at small elasticity, which may be due to its large dissipation.

As the fluid layer in this paper has an inclined temperature gradient, we can make a comparison with the pure natural convection with inclined temperature gradient of Newtonian fluid [26-28]. In the vertical direction, the temperature at the upper boundary is larger than that at the bottom in Ref. [28] and our work (see Fig. 1), which is opposite to the those in Refs. [26,27]. The liquid layer is inside two rigid walls in Refs. [26-28]. The symmetry of boundary conditions lead to the symmetry and antisymmetry in the basic flow and perturbation mode. However, the liquid layer has a free surface in this paper. There are no symmetry properties in the basic flow or perturbation mode. Meanwhile, although many modes are excited in the presence of gravity in our paper, the mode in the spanwise direction is stationary while the mode in the streamwise direction is oscillatory. In contrast, there are both oscillatory and stationary modes in streamwise and spanwise directions in Refs. [26-28]. The effect of gravity is different for the natural convection and the thermocapillary convection. It is the driving force for the former. However, the latter is driven by the thermocapillary force, while the effect of gravity is coupled with elasticity and thermocapillary force. Physically, the free surface reduces the constraint for the perturbation and the thermocapillary force becomes an important energy source. These lead to the difference of instability between the thermocapillary convection and natural convection.

We compare the results with the parameters in the experiment of drying of a polymer solution [14], where the polymer/solvent solution used is polyisobutylene/toluene. As the thermophysical properties of polymer solution depend on the polymer concentration, we estimate the parameters as follows. The Prandtl number $O(P r)$ can change from 10 to $10^{3}$, the density $O(\rho)=10^{3} \mathrm{~kg} / \mathrm{m}^{3}$, the negative rate of change of surface tension with temperature $O(\gamma)=10^{-4} \mathrm{~N} / \mathrm{m} \cdot / \mathrm{K}$, the thermal expansion $O(a)=10^{-3} \mathrm{~K}$, the thermal diffusivity $O(\chi)=10^{-7} / \mathrm{m}^{2}$. s. If we set the temperature gradient on the surface $O(b)=1 \mathrm{~K} / \mathrm{m}$ and the depth of the layer $O(d)=10^{-2} \mathrm{~m}$, the relative change of density in the vertical direction is order $10^{-5}$, so the condition for Boussinesq's approximation is satisfied. Then the Bond number $O(B o)=1$ and the Marangoni number $O(M a)=10^{3}$, which are close to the results in Fig. 2 .

\section{Conclusion}

Comparing with the flow without gravity, we can see that the gravity always destabilizes the flow for $\operatorname{Pr}=0.02$ while it often makes the flow more stable for $P r=100$. The variation of critical Marangoni number is obvious when $O(B o)=1$ for $\operatorname{Pr}=100$, $\varepsilon \leqslant 0.1$, while in other cases we studied, the corresponding Bond number has $O(B o)=10$. When the Bond number increases, the coupling of gravity to elasticity and thermocapillary force excites many different kinds of preferred modes for $P r=100$. However, the changes of preferred mode for $\operatorname{Pr}=0.02$ are far less than those for $\operatorname{Pr}=100$.

The influence of gravity for the perturbation field mainly lies in the case of spanwise stationary mode at $\mathrm{Pr}=100$ with large Bo. Its critical wave number is larger than zero and its temperature fluctuates in the vertical direction.

Energy analysis shows that the gravity becomes a new energy source for perturbation. For $\operatorname{Pr}=100$, the work done by gravity $G$ can be either positive or negative. The sign of $G$ is not directly related to the variation of $M a_{c}$ with $B o$, as $G$ can be positive (negative) when $M a_{c}$ increases (decreases) with $B$, and can even be close to zero when $O(B o)=1$. For $P r=0.02, G$ is always positive for oblique wave and spanwise stationary mode, while the energy mechanism for the streamwise wave is dominant by elasticity.

\section{Conflict of interest statement}

We declare that we have no financial and personal relationships with other people or organizations that can inappropriately influence our work, there is no professional or other personal interest of any nature or kind in any product, service and/or company that could be construed as influencing the position presented in, or the review of, the manuscript entitled.

\section{Acknowledgments}

This work has been supported by the National Natural Science Foundation of China (11402271, 11532015 and U1738119), and sponsored by K.C. Wong Magna Fund in Ningbo University.

\section{Appendix A. The perturbation equations and the Chebyshev- collocation method}

The linearized perturbation equations can be derived as follows.

$\mathrm{i} \alpha \widehat{u}+\mathrm{i} \beta \hat{v}+\widehat{w}^{\prime}=0$,

$R\left(\sigma \widehat{u}+\widehat{w} u_{0}^{\prime}+u_{0} \mathrm{i} \alpha \widehat{u}\right)=-\mathrm{i} \alpha \widehat{p}+\mathrm{i} \alpha \hat{Q}_{11}+\mathrm{i} \beta \hat{Q}_{12}+\hat{Q}_{13}^{\prime}$,

$R\left(\sigma \widehat{v}+u_{0} \mathrm{i} \alpha \widehat{v}\right)=-\mathrm{i} \beta \widehat{p}+\mathrm{i} \alpha \hat{Q}_{12}+\mathrm{i} \beta \hat{Q}_{22}+\hat{\mathrm{Q}}_{23}^{\prime}$,

$R\left(\sigma \widehat{w}+u_{0} \mathrm{i} \alpha \widehat{w}\right)=-\widehat{p}^{\prime}+\mathrm{i} \alpha \hat{Q}_{13}+\mathrm{i} \beta \hat{Q}_{23}+\hat{Q}_{33}^{\prime}+B o \cdot \widehat{T}$,

$M a\left(\widehat{u} \frac{\partial T_{0}}{\partial x}+\widehat{w} \frac{\partial T_{0}}{\partial z}+u_{0} \mathrm{i} \alpha \widehat{T}\right)+\left(\alpha^{2}+\beta^{2}\right) \widehat{T}-\widehat{T}^{\prime \prime}=-\sigma M a \widehat{T}$,

$\left(1+\lambda\left(u_{0} \mathrm{i} \alpha\right)\right) \hat{Q}_{11}-\left(1+\eta\left(u_{0} \mathrm{i} \alpha\right)\right) 2 \mathrm{i} \alpha \widehat{u}-\lambda u_{0}^{\prime} 2 \hat{Q}_{13}$

$+\eta u_{0}^{\prime}\left(2 \widehat{u^{\prime}}+2 \mathrm{i} \alpha \widehat{w}\right)+\eta u_{0}^{\prime} 2 \widehat{u^{\prime}}+\lambda\left(\widehat{w} u_{0}^{\prime \prime} \cdot u_{0}^{\prime} 4(\lambda-\eta)\right.$

$\left.-u_{0}^{\prime}\left(4(\lambda-\eta) u_{0}^{\prime} \mathrm{i} \alpha \widehat{u}+2 \widehat{u^{\prime}}\right)\right)=\sigma\left(\eta 2 \mathrm{i} \alpha \widehat{u}-\lambda \hat{Q}_{11}\right)$,

$\left(1+\lambda\left(u_{0} \mathrm{i} \alpha\right)\right) \hat{Q}_{12}-\left(1+\eta\left(u_{0} \mathrm{i} \alpha\right)\right)(\mathrm{i} \alpha \widehat{v}+\mathrm{i} \beta \widehat{u})-\lambda u_{0}^{\prime} \hat{Q}_{23}$

$+\eta u_{0}^{\prime}\left(\mathrm{i} \beta \widehat{w}+\widehat{v}^{\prime}\right)+\eta u_{0}^{\prime} \widehat{v}^{\prime}+\lambda\left(-u_{0}^{\prime}\left(2(\lambda-\eta) u_{0}^{\prime} \mathrm{i} \alpha \widehat{v}+\widehat{v}^{\prime}\right)\right)$

$=\sigma\left(\eta(\mathrm{i} \alpha \widehat{v}+\mathrm{i} \beta \widehat{u})-\lambda \hat{\mathrm{Q}}_{12}\right)$,

$$
\begin{aligned}
(1 & \left.+\lambda\left(u_{0} \mathrm{i} \alpha\right)\right) \hat{Q}_{13}-\left(1+\eta\left(u_{0} \mathrm{i} \alpha\right)\right)\left(\widehat{u}^{\prime}+\mathrm{i} \alpha \widehat{w}\right)-\lambda u_{0}^{\prime} \hat{Q}_{33}+\eta u_{0}^{\prime}\left(2 \widehat{w}^{\prime}\right) \\
& -\eta\left(\widehat{w} u_{0}^{\prime \prime}-u_{0}^{\prime}\left(\widehat{w^{\prime}}+\mathrm{i} \alpha \widehat{u}\right)\right) \\
& +\lambda\left(\widehat{w} u_{0}^{\prime \prime}-u_{0}^{\prime}\left(2(\lambda-\eta) u_{0}^{\prime} \mathrm{i} \alpha \widehat{w}+\widehat{w}^{\prime}+\mathrm{i} \alpha \widehat{u}\right)\right) \\
& =\sigma\left(\eta\left(\widehat{u^{\prime}}+\mathrm{i} \alpha \widehat{w}\right)-\lambda \hat{Q}_{13}\right),
\end{aligned}
$$

$\left(1+\lambda\left(u_{0} \mathrm{i} \alpha\right)\right) \hat{\mathrm{Q}}_{22}-\left(1+\eta\left(u_{0} \mathrm{i} \alpha\right)\right)(2 \mathrm{i} \beta \widehat{v})=\sigma\left(\eta(2 \mathrm{i} \beta \widehat{v})-\lambda \hat{Q}_{22}\right)$,

$$
\begin{aligned}
& \left(1+\lambda\left(u_{0} i \alpha\right)\right) \hat{Q}_{23}-\left(1+\eta\left(u_{0} \mathrm{i} \alpha\right)\right)\left(\mathrm{i} \beta \widehat{w}+\widehat{v}^{\prime}\right) \\
& \quad-\lambda u_{0}^{\prime} \mathrm{i} \alpha \widehat{v}+\eta u_{0}^{\prime} \mathrm{i} \alpha \widehat{v}=\sigma\left(\eta\left(\mathrm{i} \beta \widehat{w}+\widehat{v}^{\prime}\right)-\lambda \hat{Q}_{23}\right), \\
& \left(1+\lambda\left(u_{0} \mathrm{i} \alpha\right)\right) \hat{Q}_{33}-\left(1+\eta\left(u_{0} \mathrm{i} \alpha\right)\right)\left(2 \widehat{w}^{\prime}\right) \\
& \quad-\lambda u_{0}^{\prime} 2 \mathrm{i} \alpha \widehat{w}+\eta u_{0}^{\prime} 2 \mathrm{i} \alpha \widehat{w}=\sigma\left(\eta\left(2 \widehat{w}^{\prime}\right)-\lambda \hat{Q}_{33}\right) .
\end{aligned}
$$


Here, $\eta=\lambda \tilde{\beta}, u_{0}^{\prime}, u_{0}^{\prime \prime}$ are the first and second derivatives of $u_{0}$ with respect to $z$, respectively. In (A.2)-(A.4), $\hat{p}$ can be eliminated and the following equations are derived.

$$
\begin{aligned}
& R\left[\beta\left(\widehat{w} u_{0}^{\prime}+u_{0} i \alpha \widehat{u}\right)-\alpha\left(u_{0} i \alpha \widehat{v}\right)\right]-\beta\left(\mathrm{i} \alpha \hat{Q}_{11}+\mathrm{i} \beta \hat{Q}_{12}+\hat{Q}_{13}^{\prime}\right) \\
& \quad+\alpha\left(\mathrm{i} \alpha \hat{Q}_{12}+\mathrm{i} \beta \hat{Q}_{22}+\hat{Q}_{23}^{\prime}\right)=-\sigma R(\beta \widehat{u}-\alpha \widehat{v}) \\
& R \alpha\left(D \widehat{w} \cdot u_{0}^{\prime}+\widehat{w} u_{0}^{\prime \prime}+u_{0}^{\prime} \cdot i \alpha \widehat{u}+u_{0} i \alpha \widehat{u^{\prime}}\right)+R \beta\left(u_{0}^{\prime} \cdot i \alpha \widehat{v}+u_{0} i \alpha \widehat{v}^{\prime}\right) \\
& \quad-\quad R i k^{2}\left(u_{0} i \alpha \widehat{w}\right)-\left(i \alpha^{2} \hat{Q}_{11}^{\prime}+2 i \alpha \beta \hat{Q}_{12}^{\prime}+\alpha \hat{Q}_{13}^{\prime \prime}+i \beta^{2} \hat{Q}_{22}^{\prime}+\beta \hat{Q}_{23}^{\prime \prime}\right) \\
& \quad+i k^{2}\left(i \alpha \hat{Q}_{13}+i \beta \hat{Q}_{23}+\hat{Q}_{33}^{\prime}+B o \cdot \widehat{T}\right)=-\sigma R\left(\alpha \widehat{u}^{\prime}+\beta \widehat{v}^{\prime}-i k^{2} \widehat{w}\right),
\end{aligned}
$$

The normal-mode perturbation is assumed to vary with time in the form $\exp (\sigma t)$. The eigenvalue $\sigma$ can be determined by the governing Eqs. (A.1), (A.5)-(A.13) and the boundaries conditions (2.13). So there is no need for initial condition.

We use Chebyshev-collocation method [32] to obtain the perturbations. $N_{c}$ Chebyshev-collocation points $z=$ $\left(1-\cos \left(\frac{j \pi}{N_{c}+1}\right)\right) / 2, j=1-N_{c}$ are set in the flow region for the governing Eqs. (A.1), (A.5)-(A.13) while 2 points $z=0,1$ are set on the boundaries for Eqs. (2.13). The perturbation quantities are expanded in Chebyshev polynomials. For example, $\widehat{u}$ is expanded as

$\widehat{u}=\sum_{k=1}^{N_{c}+1} a_{k} H_{k-1}(\widehat{z})$

Here $\widehat{z}=1-2 z, H_{k-1}(\widehat{z})=\cos \left((k-1) \cos ^{-1}(\widehat{z})\right)$ is the $(k-1)$-th Chebyshev polynomial and $a_{k}$ is the coefficient. The general eigenvalue problem can be derived in the form of $\mathbf{W g}=\sigma \mathbf{Z g}$, where $\mathbf{W}, \mathbf{Z}$ are two matrices, $\mathbf{g}$ is the eigenvector. Then the eigenvalues can be obtained by using the $\mathrm{QZ}$ algorithm available in the Matlab-software package [32] and $N_{c}=70-90$ Chebyshev nodes are used to ensure the accuracy.

\section{References}

[1] T. DebRoy, S.A. David, Physical processes in fusion welding, Rev. Mod. Phys. 67 (1995) 85-112.

[2] T. Dufar (Ed.), Crystal Growth Processes Based on Capillarity: Czochralski, Floating Zone, Shaping and Crucible Techniques, John Wiley \& Sons, 2010.

[3] M.K. Smith, S.H. Davis, Instabilities of dynamic thermocapillary liquid layers. Part 1. Convective instabilities, J. Fluid Mech. 132 (1983) 119-144.

[4] R.J. Riley, G.P. Neitzel, Instability of thermocapillary-buoyancy convection in shallow layers. Part 1 . Characterization of steady and oscillatory instabilities, J. Fluid Mech. 359 (1998) 143-164.

[5] Y.R. Li, N. Imaishi, T. Azami, T. Hibiya, Three-dimensional oscillatory flow in a thin annular pool of silicon melt, J. Cryst. Growth 260 (2004) 28-42.

[6] J.M. Garr-Peters, The neutral stability of surface-tension driven cavity flows subject to buoyant forces. Part 1. Transverse and longitudinal disturbances, and Part 2. Oblique disturbances, Chem. Eng. Sci. 47 (1992) 1247-1276.
[7] P.M. Parmentier, V.C. Regnier, G. Lebon, Buoyant-thermocapillary instabilities in medium Prandtl-number fluid layers subject to a horizontal temperature gradient, Int. J. Heat Mass Transfer 36 (1993) 2417-2427.

[8] J.F. Mercier, C. Normand, Buoyant-thermocapillary instabilities of differentially heated liquid layers, Phys. Fluids 8 (1996) 1433-1445.

[9] F. Daviaud, J.M. Vince, Traveling waves in a fluid layer subjected to a horizontal temperature gradient, Phys. Rev. E 48 (1993) 4432.

[10] C.L. Chan, C.F. Chen, Effect of gravity on the stability of thermocapillary convection in a horizontal fluid layer, J. Fluid Mech. 647 (2010) 91-103.

[11] D.A. Nield, Surface tension and buoyancy effects in cellular convection, J. Fluid Mech. 19 (1964) 341-352.

[12] E.B. Guto, E.D. Cohen, G.I. Kheboian, Coating and Drying Defects, Wiley, New York, 1995.

[13] J.J. Chen, J.D. Lin, Thermocapillary effect on drying of a polymer solution under non-uniform radiant heating, Int. J. Heat Mass Transfer 43 (2000) 2155-2175.

[14] G. Toussaint, H. Bodiguel, F. Doumenc, B. Guerrier, C. Allain, Experimental characterization of buoyancy-and surface tension-driven convection during the drying of a polymer solution, Int. J. Heat Mass Transfer 51 (2008) 42284237.

[15] J.P. Singer, S.E. Kooi, E.L. Thomas, Focused laser-induced marangoni dewetting for patterning polymer thin films, J. Polym. Sci. Pol. Phys. 54 (2016) 225-236.

[16] Z.M. Tang, W.R. Hu, Rheological flow from a die and painting on a moving solid wall, Sci. China Phys. Mech. Astron. 47 (2004) 121-128.

[17] J.P. Downey, J.A. Pojman, Polymer Research in Microgravity: Polymerization and Processing, American Chemical Society, Washington, D.C., 2001.

[18] P.N. Kaloni, J.X. Lou, On the convective stability of Oldroyd B fluid subject to a horizontal temperature gradient, in: ASME/JSME 2003 4th Joint Fluids Summer Engineering Conference, Honolulu, Hawaii, USA, 6-10 July 2003, American Society of Mechanical Engineers, 2003, pp. 1601-1606.

[19] L.A. Davalos-Orozco, A.E. Chavez, Thermocapillary convection in a viscoelastic fluid layer under a horizontal temperature gradient, J. Appl. Polym. Sci. 49 (1991) 141-153.

[20] M.X. Tong, L.J. Yang, Q.F. Fu, Thermocapillar instability of a two-dimensional viscoelastic planar liquid sheet in surrounding gas, Phys. Fluids 26 (2014) 033105.

[21] I.J. Hernández-Hernández, L.A. Dávalos-Orozco, Competition between stationary and oscillatory viscoelastic thermocapillary convection of a film coating a thick wall, Int. J. Therm. Sci. 89 (2015) 164-173.

[22] K.X. Hu, M. He, Q.S. Chen, Instability of thermocapillary liquid layers for Oldroyd-B fluid, Phys. Fluids 28 (2016) 033105.

[23] G.T. Dee, B.B. Sauer, The surface tension of polymer liquids, Adv. Phys. 47 (2) (1998) $161-205$

[24] K.R. Rajagopal, R.K. Bhatnagar, Exact solutions for some simple flows of an Oldroyd-B fluid, Acta Mech. 113 (1) (1995) 233-239.

[25] R. Sureshkumar, A. Beris, Linear stability analysis of viscoelastic Poiseuille flow using an Arnoldi-based orthogonalization algorithm, J. Non-Newton. Fluid Mech. 56 (1995) 151-182.

[26] A.S. Ortiz-Pérez, L.A. Dávalos-Orozco, Convection in a horizontal fluid layer under an inclined temperature gradient, Phys. Fluids 23 (2011) 084107.

[27] A.S. Ortiz-Pérez, L.A. Dávalos-Orozco, Convection in a horizontal fluid layer under an inclined temperature gradient for Prandtl numbers $\operatorname{Pr}>1$, Int. J. Heat Mass Transfer 68 (2014) 444-455.

[28] A.S. Ortiz-Pérez, L.A. Dávalos-Orozco, Convection in a horizontal fluid layer under an inclined temperature gradient with a negative vertical Rayleigh number, Int. J. Heat Mass Transfer 90 (2015) 1214-1220.

[29] K.X. Hu, J. Peng, K.Q. Zhu, The linear stability of plane Poiseuille flow of Burgers fluid at very low Reynolds numbers, J. Non-Newton. Fluid Mech. 167-168 (2012) 87-94.

[30] K.X. Hu, J. Peng, K.Q. Zhu, Linear stability of plane creeping Couette flow for Burgers fluid, Acta Mech. Sin. 29 (2013) 12-23.

[31] M. Wanschura, V.M. Shevtsova, H.C. Kuhlmann, HJ. Rath, Convective instability mechanisms in thermocapillary liquid bridges, Phys. Fluids 7 (1995) 912-925.

[32] P.J. Schmid, D.S. Henningson, Stability and Transition in Shear Flows, SpringerVerlag, New York, 2001. 